\title{
Selectively reduced contrast sensitivity in high schizotypy
}

\author{
Lauren Harper $^{1} \cdot$ Emily Spencer $^{1} \cdot$ Colin Davidson $^{2} \cdot$ Claire V. Hutchinson $^{1} \mathbb{C}$
}

Received: 23 March 2019 / Accepted: 15 November 2019 / Published online: 28 November 2019

(c) The Author(s) 2019

\begin{abstract}
Deficits in the ability to encode small differences in contrast between adjacent parts of an image (contrast sensitivity) are well documented in schizophrenic patients. In the present study, we sought to determine whether contrast sensitivity deficits reported in schizophrenic patients are also evident in those who exhibit high schizotypy scores in a typical (i.e., nonschizophrenic) population. Using the O-Life Questionnaire, we determined the effects of schizotypy on spatial (0.5, 2 and 8 $\mathrm{c} / \mathrm{deg})$ and spatiotemporal $(0.5$ and $8 \mathrm{c} / \mathrm{deg}$ at 0.5 and $8 \mathrm{~Hz})$ contrast sensitivity in 73 young (18-26 years), majority female $(n=68)$ participants. We found differences in contrast sensitivity that were spatial, spatiotemporal and O-Life subscale specific. Spatial contrast sensitivity was significantly lower in high, compared to low schizotypes at low spatial frequencies $(0.5 \mathrm{c} / \mathrm{deg})$ in those who scored highly on the Unusual Experiences and Cognitive Disorganisation O-Life subscales. For moving stimuli, individuals with high scores on the Unusual Experiences subscale exhibited lower spatiotemporal contrast sensitivity for 0.5 and $8 \mathrm{c} / \mathrm{deg}$ patterns drifting at $8 \mathrm{~Hz}$. Although the effects reported here were relatively small, this is the first report of reduced contrast sensitivity in schizotypy.
\end{abstract}

Keywords Vision $\cdot$ Spatial frequency $\cdot$ Temporal frequency $\cdot$ Schizotypy $\cdot$ Psychosis proneness

\section{Introduction}

It has long been known that dopamine is involved in multiple visual processes (Djamgoz et al.1997) including contrast sensitivity (Tagliati et al. 1994). This has been shown in both animal (Bodis-Wollner 1990) and human studies (Masson et al. 1993). This should be no surprise as dopamine is found in abundance in the retina (Brandies and Yehuda 2008) and dopamine neurons innervate visual cortex (Jacob and Nienborg 2018). Visual problems are characteristic of neurodegenerative diseases known to affect the dopamine system. Parkinson's patients, for example, whose cardinal neuropathology is degeneration of the dopamine system, exhibit reduced contrast sensitivity, even in the early stages

Communicated by Melvyn A. Goodale.

Claire V. Hutchinson

ch190@1e.ac.uk

1 Department of Neuroscience, Psychology and Behaviour, College of Life Sciences, University of Leicester, Leicester, UK

2 School of Pharmacy and Biomedical Sciences, Faculty of Clinical and Biomedical Sciences, University of Central Lancashire, Preston, UK of Parkinson's Disease (Ming et al. 2016). Furthermore, contrast sensitivity deficits appear to be associated with cognitive deficits in this patent group (Ridder et al. 2017).

Schizophrenia represents another example of a wellknown brain disorder characterised by a dysfunctional dopamine system (Howes et al. 2017), although it may also involve dysfunction in serotonin, GABA and glutamate systems (Yang and Tsai 2017). Dysfunctional visual attention, cognition and executive processing are well documented in schizophrenia (Harvey et al. 2001; Barch and Ceaser 2012). Deficits in low-level visual perceptual processing have also been reported, and it has been suggested that tests of lowlevel vision should be included in diagnostic test batteries (Butler et al. 2008). The most widely studied low-level visual deficit in schizophrenia concerns the ability of schizophrenics to encode stimulus contrast. Reduced contrast sensitivity can be present even when there is no detectable impairment in visual acuity. Such reductions provide a sensitive clinical measure of visual function and can reveal abnormal visual processing at the level of the retina and in the cortical and subcortical visual pathways (Owsley 2003). Schizophrenics require significantly more contrast between adjacent parts of an image to detect that they are different. 
A number of studies have assessed conventional psychophysical contrast sensitivity (i.e., thresholds for determining the presence of a luminance-defined sinusoidal grating) in schizophrenia. The majority, but not all, studies find some evidence of schizophrenia-related contrast sensitivity deficits (Slaghuis 1998, 2004; Chen et al. 1999, 2003; Keri et al. 2002; Butler et al. 2005, 2009; Cimmer et al. 2006; Calderone et al. 2013; Cadenhead et al. 2013; Shoshina et al. 2014; Samani et al. 2018). However, in studies where deficits are reported, there is variation in the spatial and temporal frequency specificity of deficits between different patient groups and across studies. As such, at present, the clinical utility of contrast sensitivity testing in schizophrenia is somewhat limited. A summary of key studies and their findings is provided in Table 1.

Some of the differences between studies may be accounted for by the nature of contrast sensitivity testing, which is typically long and relatively labour-intensive. Instructions may sometimes be difficult for schizophrenic patients to understand. In addition, even for simple conventional tasks of contrast detection, response criteria used by patients to make decisions may differ from controls and between patients.

Another issue concerns the clinical characteristics of different groups of schizophrenics across different studies, both in terms of their symptoms and medication. This is likely to represent a particular problem in interpreting the findings of the present literature given that the numbers of schizophrenic patients included in studies are often relatively small. It is, therefore, difficult to make firm conclusions from individual studies. Indeed, in this context, it should be noted that schizophrenia has been suggested to be a heterogeneous condition; an umbrella term for a variety of overlapping conditions (Franzek and Beckmann 1998; Ban 2004). Further, the medications taken for schizophrenia might also contribute to variations amongst studies. For example, it has been suggested that typical antipsychotics, which tend to be antagonists at the dopamine D2 receptor, may improve contrast sensitivity in schizophrenic patients; whereas, atypical antipsychotics, where the mechanism of action is shifted towards blockade of the 5-HT2 receptor and less antagonism of the dopamine D2 receptor, may normalise it (Chen et al. 2003).

Schizotypy offers a means for understanding the aetiology of schizophrenia (Barrantes-Vidal et al. 2015). It also allows the determination of some characteristics of schizoaffective disorders, in our case, low-level visual disturbances such as reduced contrast sensitivity, in the absence of the potential confounds of medication. The term 'schizotypy' was coined by Meehl (1962) as a form of personality organisation commonly associated with an increased susceptibility to schizophrenia, although most schizotypes are not necessarily expected to develop psychosis (Barrantes-Vidal et al. 2015).
In the 'typical' population, it can present as subtle, subclinical manifestations of psychotic characteristics, often so subtle that it is undetectable (see Lenzenweger 2018 for a comprehensive review of schizotypy, schizotypic psychopathology and schizophrenia).

Although schizotypic traits often remain undetectable to others in everyday life, they are apparent from self-report measures. One of the most commonly used is The OxfordLiverpool Inventory of Feelings and Experiences (O-Life) Questionnaire (Mason and Claridge 2006). It is made up of 104 items, which produce a score for each participant across 4 subscales: Unusual Experiences, Cognitive Disorganisation, Introverted Anhedonia and Impulsive Nonconformity. High scores on the unusual experiences subscale can manifest as perceptual aberrations, magical thinking, and hallucinations. In the context of psychosis, the unusual experiences subscale is phenomenologically related to positive symptoms. High scores on the cognitive disorganisation subscale reflect poor attention, concentration, and decision-making, and, in the context of psychosis, are phenomenologically related to thought disorder. High scores on the introverted anhedonia subscale reflect negative schizotypy, and manifest as lack of enjoyment, withdrawal and avoidance of intimacy. High scores on the impulsive nonconformity subscale reflect anti-social, and eccentric forms of behaviour, suggestive of a lack of self-control/inhibition and asocial behaviour.

In the present study, across 3 experiments, we sought to determine the existence of a relationship between schizotypy scores on the O-Life Questionnaire and spatial and temporal contrast sensitivity. The combination of spatial and temporal frequencies used was based on previous studies of reduced contrast sensitivity in schizophrenia and schizoaffective personality disorder. Experiment 1 investigated the effects of high and low schizotypy on spatial contrast sensitivity at spatial frequencies of $0.5,2$ and $8 \mathrm{c} / \mathrm{deg}$. Experiment 2 investigated the effects of high and low schizotypy on contrast sensitivity for patterns $(0.5$ and $8 \mathrm{c} / \mathrm{deg})$ drifting at low temporal frequencies $(0.5 \mathrm{~Hz})$. Experiment 3 investigated the effects of high and low schizotypy on contrast sensitivity patterns $(0.5$ and $8 \mathrm{c} / \mathrm{deg})$ drifting at high temporal frequencies $(8 \mathrm{~Hz})$.

\section{Materials and methods}

\section{Participants}

An opportunity sample of 73 participants (68 females, 5 males), aged 18-26 years (mean 19.5 years; SD 1.8) took part in the study. Participants were undergraduate students in the School of Psychology at the University of Leicester. They had no history of ocular disease and reported that they were not taking any medications at the time of testing. 


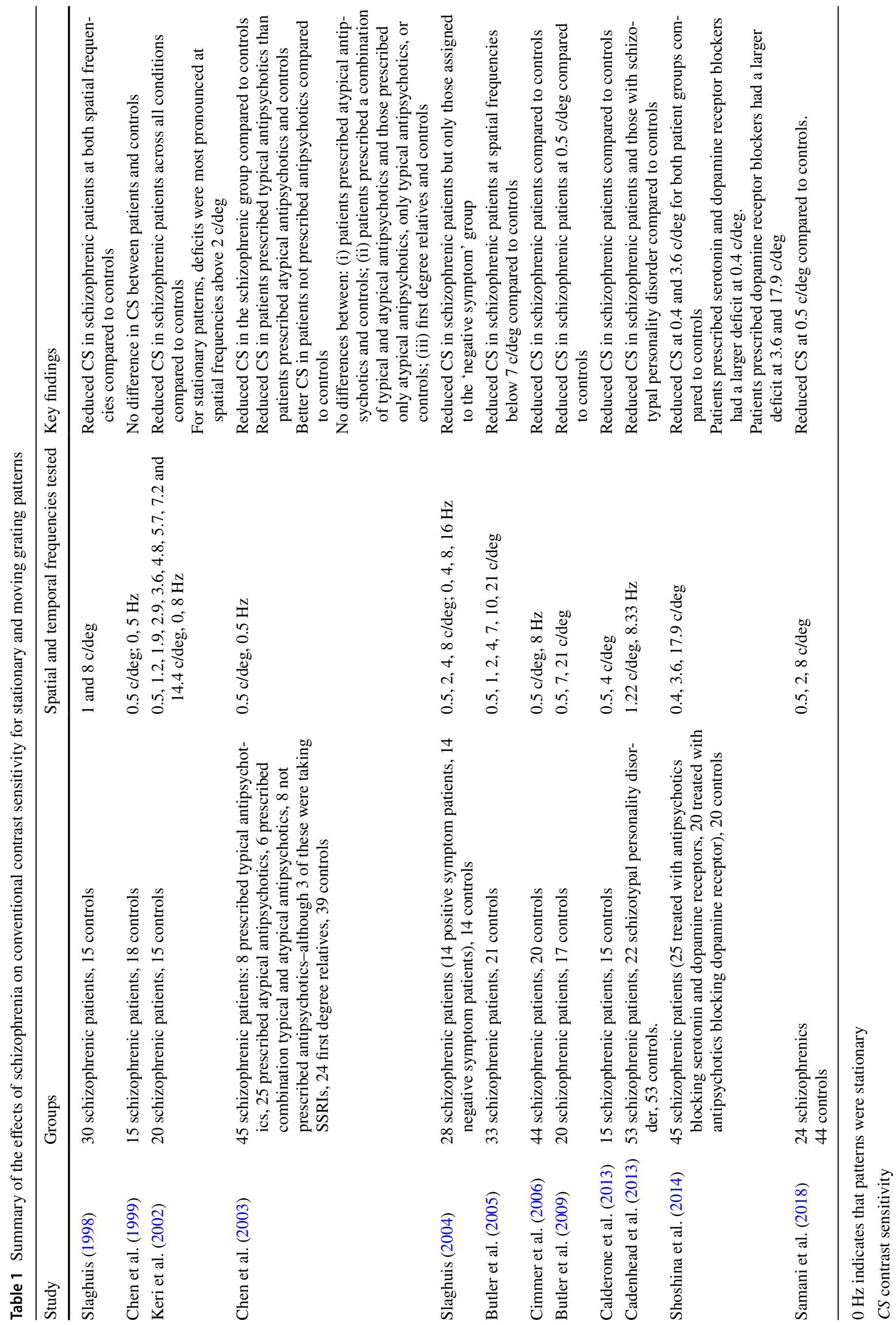


Binocular corrected visual acuity at near and distance was in the normal range for all participants included in the study. Ethical approval was granted by the University of Leicester. All experimental methods adhered to the tenets of the Declaration of Helsinki. Informed consent was obtained before the study commenced. Upon admission to the study, participants completed the O-Life questionnaire.

\section{Apparatus and stimuli}

Sinusoidal gratings subtended 6 degrees (horizontally and vertically) at a viewing distance of $69.5 \mathrm{~cm}$ and were generated using a Macintosh G4 and presented on a Sony Trinitron CRT monitor with an update rate of $75 \mathrm{~Hz}$ using the C programming language. The monitor was gamma-corrected using a spot photometer (LS-100, Konica Minolta) and lookup-tables (LUT). For precise control of luminance contrast, the number of intensity levels available was increased from 8 to 14 bits using a Bits ++ attenuator (Cambridge Research Systems). The mean luminance of the display was $\sim 64 \mathrm{~cd} / \mathrm{m}^{2}$ $(\min =1.16 ; \max =127.7)$ and the monitor was the only light source. Total stimulus presentation duration was $853 \mathrm{~ms}$ and the luminance contrast of the sinusoidal waveform was smoothed on and off by half a cycle of a raised cosine lasting $170 \mathrm{~ms}$. In a similar manner, the sinusoidal waveform was spatially windowed in the horizontal dimension according to a half cycle of a raised cosine function with a half-period of $1.2 \mathrm{deg}$. This was done to minimise the presence of spatial and temporal transients.

The Michelson contrast of the pattern could be varied according to the following equation:

Luminance contrast $=\left(L_{\max }-L_{\min }\right) /\left(L_{\max }+L_{\min }\right)$,

where $L_{\max }$ and $L_{\min }$ are the maximum and the minimum luminances of the grating, in the range $0-1$.

\section{Procedure}

Contrast threshold measurements were taken using a single-interval, forced-choice procedure. In Experiment 1, on each trial, participants were presented with a fixation cross, followed by the presentation of a stationary grating, upon which they were required to judge its orientation (vertical or horizontal). In Experiments 2 and 3, on each trial, participants were presented with a fixation cross, followed by the presentation of a moving pattern and required to judge its direction (left vs right). Before each experiment commenced, participants were allowed a short practice run. The luminance contrast of the test stimulus was varied from trial to trial according to a modified 3-down, 1-up staircase designed to converge on the contrast corresponding to $79.4 \%$ correct. At the beginning of each run of trials, the contrast of the test pattern was initially set to a suprathreshold level (typically $\sim 6 \mathrm{~dB}$ above threshold) and the initial staircase step size was chosen to be half of this value. On subsequent reversals, the step size was halved and testing was terminated after a total of 16 reversals. Threshold estimates were taken as the mean of the last 4 reversals in each staircase. Each observer completed 2 staircases per condition and a mean was taken. The order of testing was randomised within each experiment.

\section{Data analysis}

Median splits were performed for each O-Life subscale. ${ }^{1}$ This provided groups of high and low scorers on each subscale as follows: Unusual Experiences: $n=68$ ( 35 high, 33 low); Cognitive Disorganisation: $n=65$ (32 high, 33 low); Introverted Anhedonia: $n=63$ (34 high, 29 low); Impulsive Non-conformity: $n=62$ (33 high, 29 low). Contrast thresholds were converted to contrast sensitivity (1/contrast threshold) for graphical representations of data and statistical analyses. For Experiment 1, 3 (spatial frequency: 0.5, 2, 8 c/deg) by 2 (group: high, low), mixed, repeated measures analyses of variance were performed separately for each subscale. For Experiments 2 and 3, 2 (spatial frequency: 0.5, $8 \mathrm{c} / \mathrm{deg}$ ) by 2 (group: high, low), mixed, repeated measures analyses of variance were performed separately for each subscale. Significant findings were investigated further using post hoc independent samples $t$-tests and regression analyses.

\section{Results}

\section{Experiment 1: spatial contrast sensitivity}

Mean ( $\pm 95 \%$ confidence intervals) and median contrast thresholds at $0.5,2$ and $8 \mathrm{c} / \mathrm{deg}$ for those who scored 'high' and 'low' on each O-Life subscale are given in Table 2. Mean contrast sensitivity (1/contrast threshold) is shown in Fig. 1.3 (spatial frequency: $0.5,2,8 \mathrm{c} / \mathrm{deg}$ ) by 2 (group: high, low) analyses of variance performed separately for each subscale revealed significant main effects of spatial frequency on contrast sensitivity across all subscales as

\footnotetext{
1 There are a number of ways in which we could have split our data into 'high' and 'low' schizotypy. For example, if we had only included participants with very high and very low values, any differences we found may have been more pronounced and/or stronger interactions may have emerged between some conditions. However, the purpose of our study was to determine whether subtle differences in schizotypal personality traits are reflected in contrast sensitivity scores in a typical/general population. As such, we performed a standard median split on the data, i.e., any scores above the median were assigned to 'high' and any scores below the median were assigned to 'low'.
} 
Table 2 Mean (with lower (c) and upper (+) $95 \%$ confidence intervals) and median contrast thresholds (in the range $0-1$ ) at $0.5,2$ and $8 \mathrm{c} / \mathrm{deg}$ for high and low schizotypes on each O-Life subscale

\begin{tabular}{|c|c|c|c|c|}
\hline Spatial frequency & Subscale & Score & Mean ( $\pm 95 \%$ CIs) & Median \\
\hline \multirow[t]{8}{*}{$0.5 \mathrm{c} / \mathrm{deg}$} & \multirow[t]{2}{*}{ Unusual experiences } & High & 0.0092 (-CI: $0.0052 ;+$ CI: 0.0132 ) & 0.0057 \\
\hline & & Low & 0.0049 ( - CI: $0.0043 ;+$ CI: 0.0055$)$ & 0.0046 \\
\hline & \multirow[t]{2}{*}{ Cognitive disorganisation } & High & 0.0090 ( - CI: 0.0049; + CI:0.0132) & 0.0052 \\
\hline & & Low & 0.0049 (- CI: $0.0043 ;+$ CI:0.0056) & 0.0047 \\
\hline & \multirow[t]{2}{*}{ Introverted anhedonia } & High & 0.0075 ( - CI: $0.0043 ;+$ CI: 0.0107) & 0.0053 \\
\hline & & Low & 0.0050 ( - CI: $0.0045 ;+$ CI: 0.0055) & 0.0049 \\
\hline & \multirow[t]{2}{*}{ Impulsive nonconformity } & High & 0.0074 ( - CI: $0.0044 ;+$ CI: 0.0105) & 0.0052 \\
\hline & & Low & 0.0071 (- CI: 0.00355; + CI: 0.0108) & 0.0053 \\
\hline \multirow[t]{8}{*}{$2 \mathrm{c} / \mathrm{deg}$} & \multirow[t]{2}{*}{ Unusual experiences } & High & 0.0024 ( - CI: $0.0022 ;+$ CI: 0.0028) & 0.0023 \\
\hline & & Low & 0.0027 ( - CI: $0.0023 ;+$ CI: 0.0032 ) & 0.0024 \\
\hline & \multirow[t]{2}{*}{ Cognitive disorganisation } & High & 0.0024 ( - CI: $0.0022 ;+$ CI:0.0027) & 0.0022 \\
\hline & & Low & 0.0027 ( - CI: 0.0023; + CI:0.0032) & 0.0024 \\
\hline & \multirow[t]{2}{*}{ Introverted anhedonia } & High & 0.0026 ( - CI: $0.0022 ;+$ CI: 0.0031$)$ & 0.0024 \\
\hline & & Low & 0.0024 (- CI: $0.0022 ;+$ CI: 0.0026$)$ & 0.0024 \\
\hline & \multirow[t]{2}{*}{ Impulsive nonconformity } & High & 0.0024 ( - CI: 0.0022; + CI:0.0026) & 0.0022 \\
\hline & & Low & 0.0025 (- CI: $0.0023 ;+$ CI: 0.0028$)$ & 0.0024 \\
\hline \multirow[t]{8}{*}{$8 \mathrm{c} / \mathrm{deg}$} & \multirow[t]{2}{*}{ Unusual experiences } & High & 0.0077 ( - CI: 0.0062; + CI: 0.0093) & 0.0065 \\
\hline & & Low & 0.0098 (- CI: 0.0043; +CI: 0.0153) & 0.0058 \\
\hline & \multirow[t]{2}{*}{ Cognitive disorganisation } & High & 0.0079 ( - CI: 0.0057; + CI:0.0102) & 0.0054 \\
\hline & & Low & 0.0075 (- CI: 0.0062; +CI:0.0089) & 0.0058 \\
\hline & \multirow[t]{2}{*}{ Introverted anhedonia } & High & 0.0072 ( - CI: $0.0060 ;+$ CI: 0.0084$)$ & 0.0058 \\
\hline & & Low & 0.0109 ( - CI: $0.0043 ;+$ CI: 0.0174$)$ & 0.0058 \\
\hline & \multirow[t]{2}{*}{ Impulsive nonconformity } & High & 0.0098 ( - CI: 0.0043; + CI: 0.0152) & 0.0065 \\
\hline & & Low & 0.0079 ( - CI: 0.0054; + CI: 0.0106) & 0.0056 \\
\hline
\end{tabular}

follows: Unusual Experiences: $\mathrm{F}(1.733,114.361)=129.425$; $p<0.001 ; \eta p^{2}=0.662$; Cognitive Disorganisation: $\mathrm{F}(1.682,105.980)=122.753 ; p<0.001 ; \eta p^{2}=0.661$; Introverted Anhedonia: $\mathrm{F}(1.656,101.023)=109.160 ; p<0.001$; $\eta p^{2}=0.642$; Impulsive Non-conformity: $\mathrm{F}(2120)=242.195$; $p<0.001 ; \eta p^{2}=0.801$. There were no main effects of group for any of the subscales. There were small but significant spatial frequency $\mathrm{x}$ group interactions for the subscales Unusual Experiences $[\mathrm{F}(1.733,114.361)=4.219$; $\left.p=0.011 ; \eta p^{2}=0.060\right]$ and Cognitive Disorganisation $\left[\mathrm{F}(1.682,105.980)=3.620 ; p=0.016 ; \eta p^{2}=0.054\right]$. Post hoc, Bonferroni-corrected t-tests performed at each spatial frequency $(0.5,2,8 \mathrm{c} / \mathrm{deg})$ showed that this interaction reflected significantly lower contrast sensitivity at $0.5 \mathrm{c} / \mathrm{deg}$ in those who scored highly on the Unusual Experiences $[t=-2.278$; $\mathrm{df}=66 ; p=0.013 ; d=0.57]$ and Cognitive Disorganisation $[t=-230 ; \mathrm{df}=63 ; p=0.034 ; d=0.47]$ subscales. To provide a better representation of the distribution of contrast sensitivity scores for high and low schizotypes, for conditions on which significant differences emerged (unusual experiences and cognitive disorganisation subscales at $0.5 \mathrm{c} / \mathrm{deg}$ ), box and whisker plots are provided in Fig. 2. It is evident that, although schizotypy had a significant effect under these conditions, there was considerable overlap between groups. Further, regression analyses confirmed that individual scores on each subscale significantly predicted contrast sensitivity at low spatial frequencies (Unusual Experiences: $R^{2}=0.047, F(1,71)=3.56 p=0.031$; Cognitive Disorganisation: $\left.R^{2}=0.053, F(1,71)=3.95 p=0.026\right)$, shown in Fig. 3 .

\section{Experiment 2: spatiotemporal contrast sensitivity at low temporal frequencies}

Table 3 gives mean ( $\pm 95 \%$ confidence intervals) and median contrast thresholds for 0.5 and $8 \mathrm{c} / \mathrm{deg}$ patterns drifting at $0.5 \mathrm{~Hz}$ for those who scored 'high' and 'low' on each O-Life subscale. Mean contrast sensitivity (1/contrast threshold) is shown in Fig. 4. A 2 (spatial frequency: $0.5,8 \mathrm{c} / \mathrm{deg}$ ) by 2 (group: high, low) analysis of variance performed separately for each subscale revealed significant main effects of spatial frequency on contrast sensitivity across all subscales as follows: Unusual Experiences: $F(1,66)=207.385$; $p<0.001 ; \eta p^{2}=0.759$; Cognitive Disorganisation: $F(1,63)=214.607 ; p<0.001 ; \eta p^{2}=0.773$; Introverted Anhedonia: $F(1,61)=203.896 ; p<0.001 ; \eta p^{2}=0.770$; Impulsive Non-conformity: $\underline{F}(1,60)=195.132 ; p<0.001 ; \eta p^{2}=0.765$. There were no effects of group nor were there any spatial frequency $x$ group interactions. 


\section{(a) Unusual Experiences}

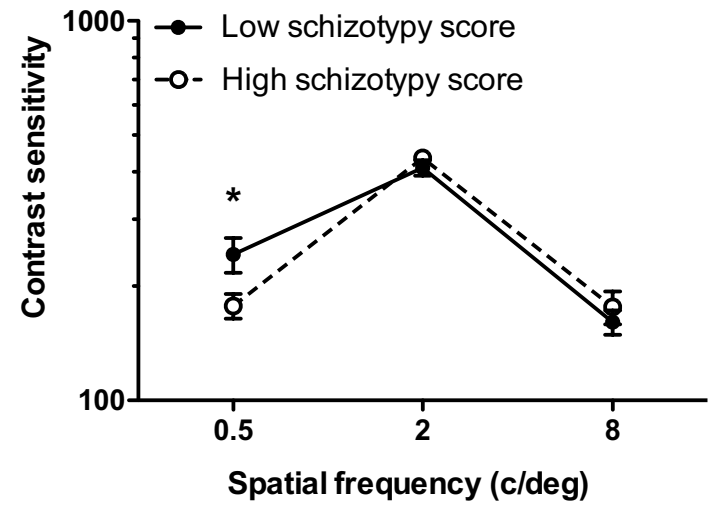

(c) Introverted Anhedonia

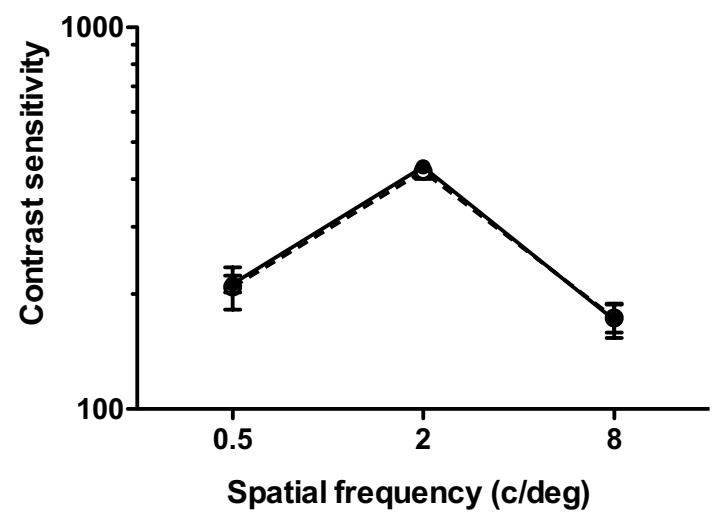

(b) Cognitive Disorganisation

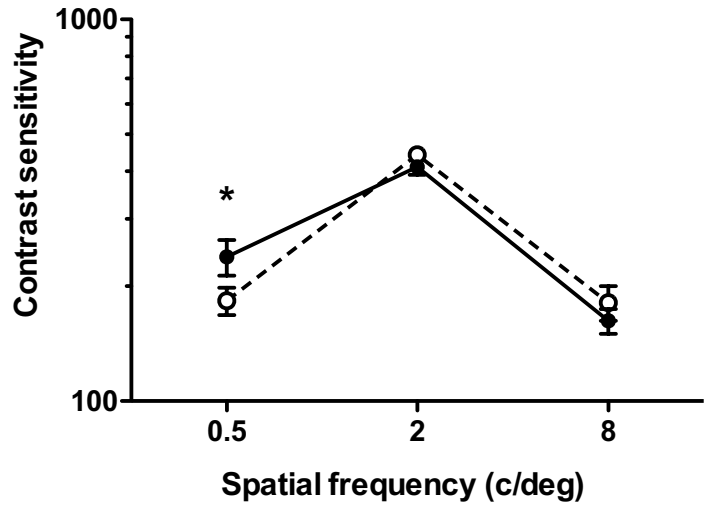

(d) Impulsive Non-conformity

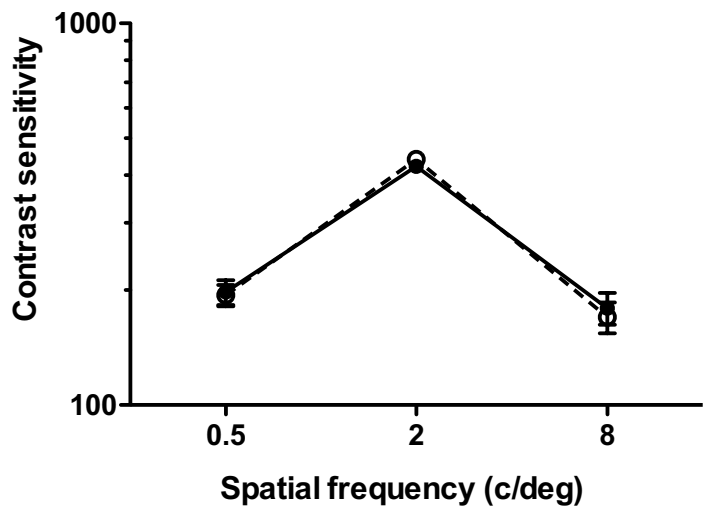

Fig. 1 Mean contrast sensitivity at 0.5, 2 and 8 c/deg for those who scored 'high' (open symbols) and 'low' (closed symbols) on each O-Life subscale: a unusual experiences, $\mathbf{b}$ cognitive disorganisation, $\mathbf{c}$ introverted anhedonia and $\mathbf{d}$ impulsive non-conformity. Error bars are \pm 1 SEM

(a) Unusual experiences

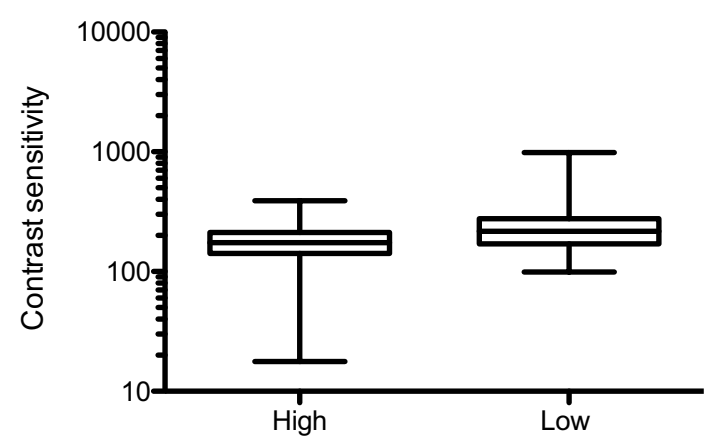

(b) Cognitive disorganisation

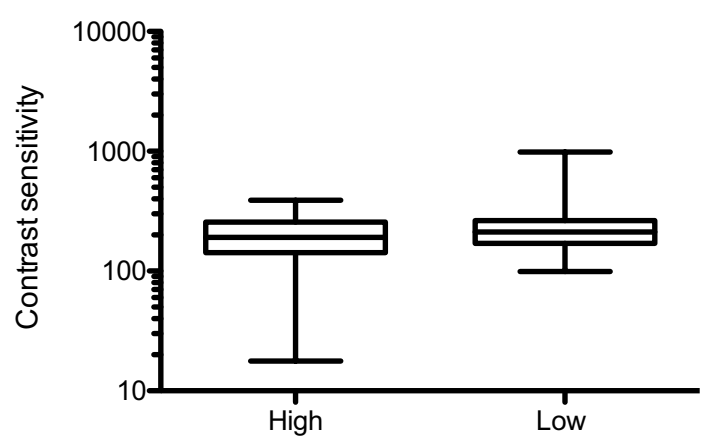

Fig. 2 Box and whisker plots showing minimum, 1st quartile, median, 3rd quartile and maximum contrast sensitivity at 0.5 c/deg for those who scored 'high' or 'low' on the a unusual experiences and b cognitive disorganisation subscales of the O-Life Questionnaire 


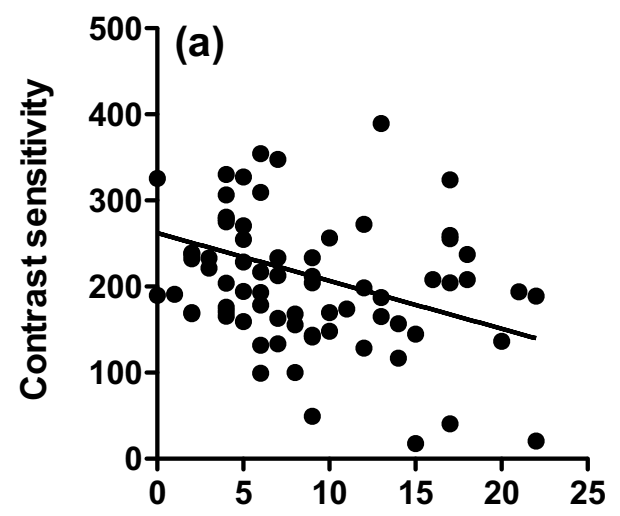

Unusual Experiences Subscale Score

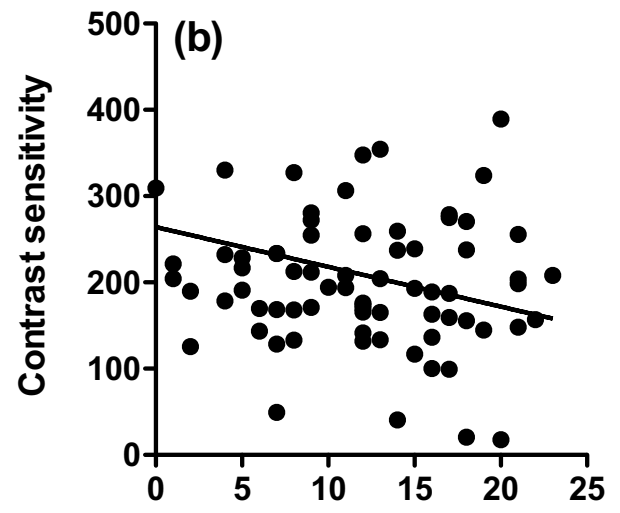

Cognitive Disorganisation Subscale Score

Fig. 3 Individual scores on the a unusual experiences and $\mathbf{b}$ cognitive disorganisation subscales of the O-Life questionnaire against contrast sensitivity scores at $0.5 \mathrm{c} / \mathrm{deg}$

Table 3 Mean (with lower (-) and upper $(+) 95 \%$ confidence intervals) and median contrast thresholds (in the range 0-1) for 0.5 and $8 \mathrm{c} / \mathrm{deg}$ patterns drifting at $0.5 \mathrm{~Hz}$ for each O-Life subscale

\begin{tabular}{|c|c|c|c|c|}
\hline Spatial frequency & Subscale & $\begin{array}{l}\text { Schi- } \\
\text { zotypy } \\
\text { group }\end{array}$ & Mean ( $\pm 95 \%$ CIs) & Median \\
\hline \multirow[t]{8}{*}{$0.5 \mathrm{c} / \mathrm{deg}$} & \multirow[t]{2}{*}{ Unusual experiences } & High & 0.0064 ( - CI: $0.0047 ;+$ CI: 0.0080$)$ & 0.0047 \\
\hline & & Low & 0.0064 ( - CI: 0.0036; + CI: 0.0091) & 0.0047 \\
\hline & \multirow[t]{2}{*}{ Cognitive disorganisation } & High & 0.0057 ( - CI: 0.0040; + CI:0.0073) & 0.0046 \\
\hline & & Low & 0.0066 ( - CI: $0.0038 ;+$ CI: 0.0094$)$ & 0.0048 \\
\hline & \multirow[t]{2}{*}{ Introverted anhedonia } & High & 0.0064 ( - CI: 0.0037; +CI: 0.0091) & 0.0047 \\
\hline & & Low & 0.0059 ( - CI: $0.0044 ;+$ CI: 0.0073$)$ & 0.0047 \\
\hline & \multirow[t]{2}{*}{ Impulsive nonconformity } & High & 0.0056 ( - CI: $0.0041 ;+$ CI: 0.0070$)$ & 0.0047 \\
\hline & & Low & 0.0058 ( - CI: $0.0042 ;+$ CI: 0.0074$)$ & 0.0046 \\
\hline \multirow[t]{8}{*}{$8 \mathrm{c} / \mathrm{deg}$} & \multirow[t]{2}{*}{ Unusual experiences } & High & 0.6604 ( - CI: $0.5271 ;+$ CI: 0.7937) & 0.8933 \\
\hline & & Low & 0.6561 ( - CI: $0.5182 ;+$ CI: 0.7940$)$ & 0.8242 \\
\hline & \multirow[t]{2}{*}{ Cognitive disorganisation } & High & 0.6138 (- CI: $0.4780 ;+$ CI: 0.7495$)$ & 0.7145 \\
\hline & & Low & 0.7565 ( - CI: $0.6299 ;+$ CI: 0.8830$)$ & 0.9663 \\
\hline & \multirow[t]{2}{*}{ Introverted anhedonia } & High & 0.6400 ( - CI: $0.4975 ;+$ CI: 0.7825 ) & 0.8612 \\
\hline & & Low & 0.6277 ( - CI: $0.4827 ;+$ CI: 0.7727 ) & 0.7732 \\
\hline & \multirow[t]{2}{*}{ Impulsive nonconformity } & High & 0.6366 (- CI: $0.4895 ;+$ CI: 0.7838 ) & 0.8933 \\
\hline & & Low & 0.6904 ( - CI: 0.5564; +CI: 0.8244) & 0.8242 \\
\hline
\end{tabular}

\section{Experiment 3: spatiotemporal contrast sensitivity at high temporal frequencies}

Table 4 gives mean ( $\pm 95 \%$ confidence intervals) and median contrast thresholds for 0.5 and $8 \mathrm{c} / \mathrm{deg}$ patterns drifting at $8 \mathrm{~Hz}$ for those who scored 'high' and 'low' on each O-Life subscale. Mean contrast sensitivity (1/contrast threshold) is shown in Fig. 5. A 2 (spatial frequency: $0.5,8 \mathrm{c} / \mathrm{deg}$ ) by 2 (group: high, low) analysis of variance performed separately for each subscale revealed significant main effects of spatial frequency on contrast sensitivity across all subscales as follows: Unusual Experiences: $F(1,66)=203.00$; $p<0.001 ; \eta p^{2}=0.755$; Cognitive Disorganisation:
$F(1,63)=202.876 ; p<0.001 ; \eta p^{2}=0.763$; Introverted Anhedonia: $F(1,61)=188.414 ; p<0.001 ; \eta p 2=0.755$; Impulsive Non-conformity: $F(1,60)=183.508 ; p<0.001 ; \eta p^{2}=0.754$. There was a small but significant, main effect of group for the subscale 'Unusual Experiences' $[F(1,66)=3.278$; $\left.p=0.037 ; \eta p^{2}=0.047\right]$, in that those categorised as 'high' schizotypes exhibited worse contrast sensitivity. There was no spatial frequency by group interaction, indicating that contrast sensitivity was significantly impaired at both spatial frequencies. The distributions of contrast sensitivity scores for high and low schizotypes on the unusual experiences O-Life subscale are shown in Fig. 6. 
(a) Unusual Experiences

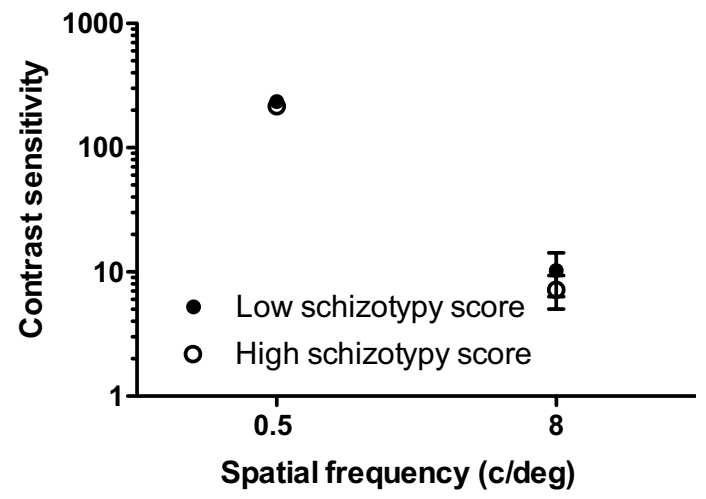

(c) Introverted Anhedonia

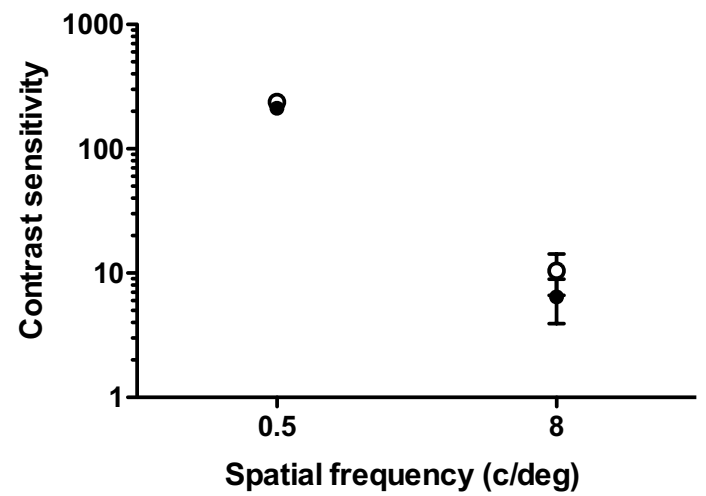

\section{(b) Cognitive Disorganisation}

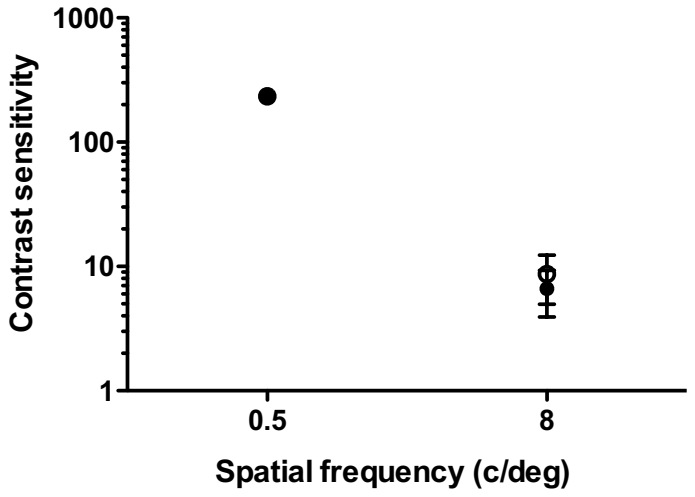

(d) Impulsive Non-conformity

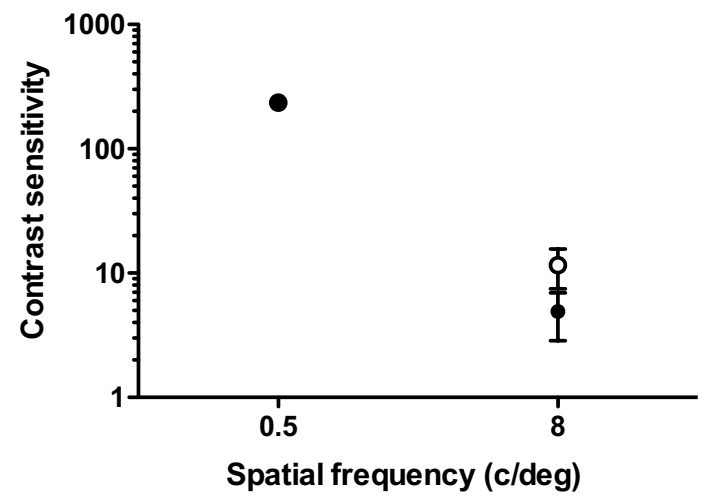

Fig. 4 Mean contrast sensitivity for 0.5 and 8 c/deg patterns drifting at $0.5 \mathrm{~Hz}$ for those who scored 'high' and 'low' on each O-Life subscale: a unusual experiences, $\mathbf{b}$ cognitive disorganisation, $\mathbf{c}$ introverted anhedonia and $\mathbf{d}$ impulsive non-conformity. Error bars are \pm 1 S.E.M

Table 4 Mean (with lower $(-)$ and upper (+) $95 \%$ confidence intervals) and median contrast thresholds (in the range $0-1$ ) for 0.5 and 8 c/deg patterns drifting at $8 \mathrm{~Hz}$ for each O-Life subscale

\begin{tabular}{|c|c|c|c|c|}
\hline Spatial frequency & Subscale & $\begin{array}{l}\text { Schi- } \\
\text { zotypy } \\
\text { group }\end{array}$ & Mean ( $\pm 95 \%$ CIs) & Median \\
\hline \multirow[t]{8}{*}{$0.5 \mathrm{c} / \mathrm{deg}$} & \multirow[t]{2}{*}{ Unusual experiences } & High & 0.013 ( - CI: 0.0004; +CI: 0.0256) & 0.0019 \\
\hline & & Low & 0.0026 (- CI: $0.0018 ;+$ CI: 0.0032 ) & 0.0019 \\
\hline & \multirow[t]{2}{*}{ Cognitive disorganisation } & High & 0.0069 (- CI: $0.0006 ;+$ CI:0.0133) & 0.0018 \\
\hline & & Low & 0.0027 (- CI: $0.0017 ;+$ CI: 0.0036$)$ & 0.0018 \\
\hline & \multirow[t]{2}{*}{ Introverted anhedonia } & High & 0.0029 (- CI: $0.0015 ;+$ CI: 0.0044$)$ & 0.0018 \\
\hline & & Low & 0.0128 (- CI: $0.0022 ;+$ CI: 0.0278) & 0.0019 \\
\hline & \multirow[t]{2}{*}{ Impulsive nonconformity } & High & 0.0099 (- CI: 0.0026; + CI: 0.0224) & 0.0018 \\
\hline & & Low & 0.0064 (- CI: 0.0002; + CI: 0.0126) & 0.0019 \\
\hline \multirow[t]{8}{*}{$8 \mathrm{c} / \mathrm{deg}$} & \multirow[t]{2}{*}{ Unusual experiences } & High & 0.1814 (- CI: 0.0648;+CI: 0.2979) & 0.0106 \\
\hline & & Low & 0.1202 (- CI: $0.0231 ;+$ CI: 0.2174$)$ & 0.0077 \\
\hline & \multirow[t]{2}{*}{ Cognitive disorganisation } & High & 0.1395 (- CI: 0.0297; +CI: 0.2493) & 0.0095 \\
\hline & & Low & 0.1131 (- CI: $0.0217 ;+$ CI: 0.2044$)$ & 0.0098 \\
\hline & \multirow[t]{2}{*}{ Introverted anhedonia } & High & 0.1144 (- CI: $0.0250 ;+$ CI: 0.2038) & 0.0092 \\
\hline & & Low & 0.1935 (- CI: 0.0604; +CI: 0.3266) & 0.0135 \\
\hline & \multirow[t]{2}{*}{ Impulsive nonconformity } & High & 0.1072 (- CI: 0.0165; + CI: 0.1979) & 0.0087 \\
\hline & & Low & 0.1677 ( - CI: 0.0469; + CI: 0.2885) & 0.0134 \\
\hline
\end{tabular}


(a) Unusual Experiences

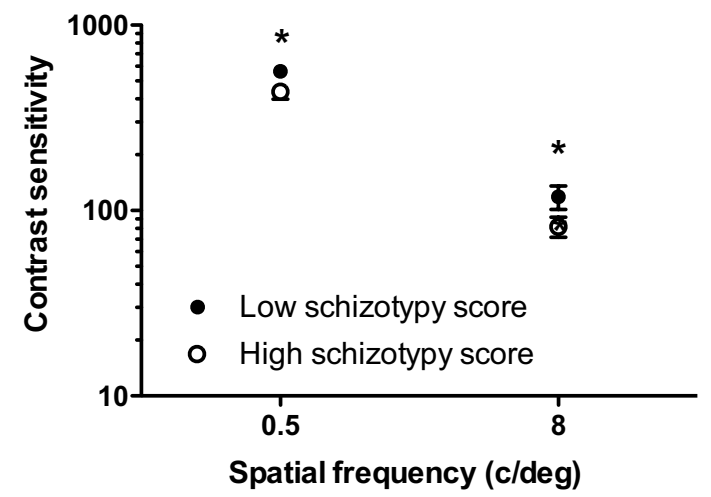

(c) Introverted Anhedonia

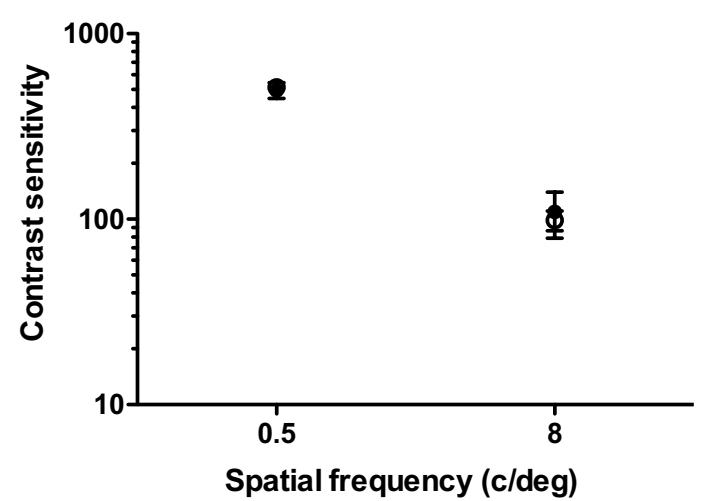

(b) Cognitive Disorganisation

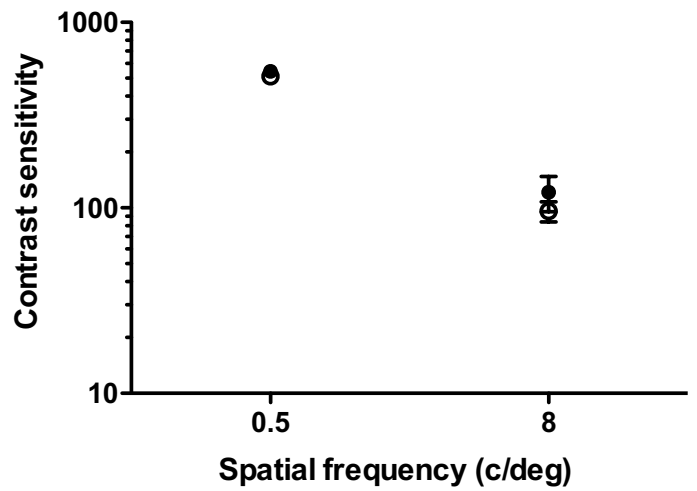

(d) Impulsive Non-conformity

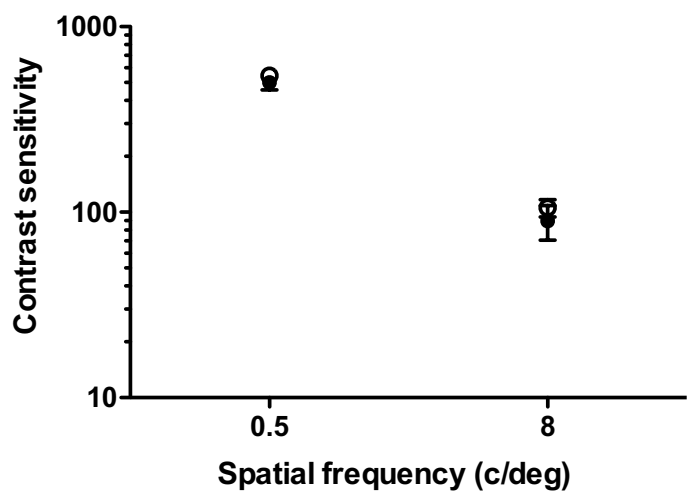

Fig. 5 Mean contrast sensitivity for 0.5 and $8 \mathrm{c} / \mathrm{deg}$ patterns drifting at $8 \mathrm{~Hz}$ for those who scored 'high' and 'low' on each O-Life subscale: a unusual experiences, $\mathbf{b}$ cognitive disorganisation, $\mathbf{c}$ introverted anhedonia and $\mathbf{d}$ impulsive non-conformity. Error bars are \pm 1 S.E.M

(a) $0.5 \mathrm{c} / \mathrm{deg}$

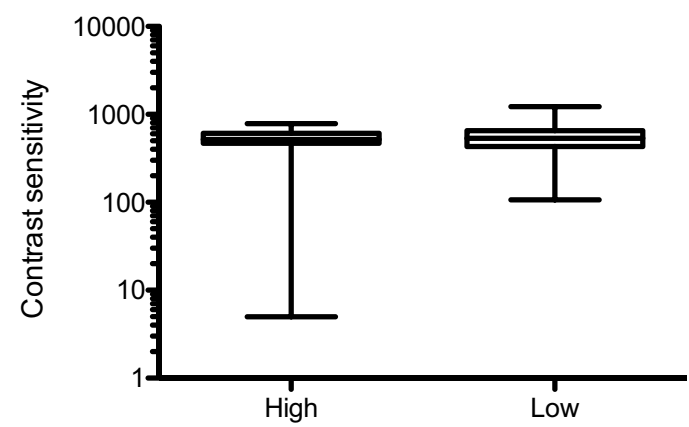

(b) $8 \mathrm{c} / \mathrm{deg}$

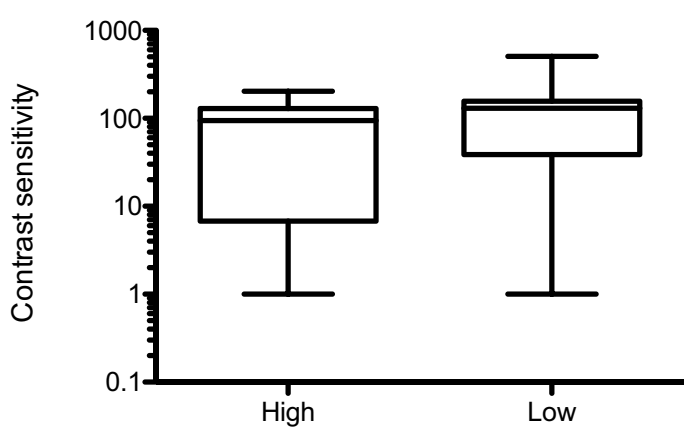

Fig. 6 Box and whisker plots showing minimum, 1st quartile, median, 3rd quartile and maximum contrast sensitivity at a $0.5 \mathrm{c} / \mathrm{deg}$ and $\mathbf{b} 8 \mathrm{c} /$ deg for patterns drfiting at $8 \mathrm{~Hz}$ in those who scored 'high' or 'low' on the unusual experiences subscale of the O-Life questionnaire 
Regression analyses did not reveal any significant associations between individual scores on the Unusual Experiences subscale and contrast sensitivity at these spatiotemporal frequencies.

\section{Discussion}

The results of the present study revealed schizotypyrelated differences in spatial and spatiotemporal contrast sensitivity. Spatial contrast sensitivity was significantly lower in high, compared to low schizotypes at low spatial frequencies $(0.5 \mathrm{c} / \mathrm{deg})$ but not intermediate $(2 \mathrm{c} / \mathrm{deg})$ or higher $(8 \mathrm{c} / \mathrm{deg})$ spatial frequencies. These differences were subscale specific, only being evident on the Unusual Experiences and Cognitive Disorganisation subscales. For moving stimuli, individuals with high scores on the Unusual Experiences subscale exhibited lower spatiotemporal contrast sensitivity at higher $(8 \mathrm{~Hz})$, but not lower $(0.5 \mathrm{~Hz})$ temporal frequencies. Scores on the introverted anhedonia and impulsive nonconformity subscales had no effect on contrast sensitivity across any spatial and/or temporal frequencies.

Deficits were most pronounced for spatial contrast sensitivity (i.e., sensitivity to the contrast of stationary patterns). These results are in keeping with studies in schizophrenia where reduced contrast sensitivity is most commonly found for stationary patterns. In terms of the spatial frequency profile of reduced contrast sensitivity, these were only evident in high schizotypes at low spatial frequencies. In schizophrenics, reduced contrast sensitivity has been shown to selectively affect low to intermediate spatial frequencies (Butler et al. 2005, 2009; Shoshina et al. 2014; Samani et al. 2018). However, other studies have found more nonselective spatial contrast sensitivity across a range of spatial frequencies (Slaghuis 1998; Keri et al. 2002; Chen et al. 2003), whereas others find no deficit (Chen et al. 1999). For moving patterns, we found selectively reduced contrast sensitivity at high $(8 \mathrm{~Hz})$, but not low $(0.5 \mathrm{~Hz})$ temporal frequencies, irrespective of the spatial frequencies $(0.5,8 \mathrm{c} /$ deg) we used. In schizophrenia, reduced contrast sensitivity for moving test patterns has been shown in some (Keri et al. 2002; Chen et al. 2003; Slaghuis 2004; Cimmer et al. 2006; Cadenhead et al. 2013), but not all (Chen et al. 1999) schizophrenic groups across temporal frequencies ranging from 0.5 to $16 \mathrm{~Hz}$. Improved contrast sensitivity has also been documented in schizophrenia under some conditions (Chen et al. 2003). Our findings at high temporal frequencies might also reflect increased internal noise (decreased signal-noise ratios) in those who exhibited high schizotypy scores. In the context of schizophrenia, a recent study (Chen et al. 2014) using random dot kinematograms has shown that schizophrenics exhibited significantly poorer speed discrimination across a range of speeds (5.25-13.0 deg/s) and their performance was more susceptible to the addition of noise compared to controls.

One finding of note is that, even for spatial contrast sensitivity, deficits were only apparent in individuals who scored highly on the unusual experiences and cognitive disorganisation subscales of the O-Life questionnaire. For moving patterns, deficits at high temporal frequencies were only apparent for those who scored highly on the unusual experiences subscales. That reduced contrast sensitivity in high schizotypes was subscale specific may be accounted for by the nature of the schizotypic characteristics comprised within each subscale. High scores on the unusual experiences subscale manifest as perceptual aberrations, magical thinking, and hallucinations, commonly associated with positive symptoms of schizophrenia. The characteristics comprised in this subscale are considerably less subtle than those represented in other subscales (e.g., the lack of enjoyment and withdrawal and avoidance of intimacy encapsulated by the introverted anhedonia subscale, and the components of anti-social and eccentric behaviour in the impulsive nonconformity subscale). That reduced spatial contrast sensitivity was evident in those who scored highly on the cognitive disorganisation scales, represented by poor attention, concentration, and decisionmaking, are in keeping with other studies that consistently document difficulties with attention and cognition in both high schizotypes and schizophrenics (Luck and Gold 2008; Ettinger et al. 2015).

One possible caveat of the findings presented here is that our sample was restricted to young adults, the majority of whom were female. As such, it may be that our findings would not necessarily generalise beyond a sample of this type. For example, using the Schizotypal Personality Questionnaire (SPQ), Bora and Baysan Arabaci (2009) have presented evidence that some schizotypal personality traits may be most prevalent in younger adults, becoming less pronounced with age. Of particular relevance to the present study is the finding that, compared to older age groups, younger participants exhibited higher scores on the unusual perceptual experiences subscale of the SPQ. Some gender differences in schizotypal characteristics have also been reported. Where male participants tend to exhibit higher scores than female participants on disorganised and negative symptom-like aspects of schizotypy (Mata et al. 2005; Bora and Baysan Arabaci 2009), female participants tend to exhibit higher scores on scale items related to social anxiety, magical thinking, paranoia and odd beliefs (Mata et al. 2005; Fonseca-Pedrero et al. 2008; Bora and Baysan Arabaci 2009).

In conclusion, we have shown reduced contrast sensitivity in high schizotypy for stationary and moving test patterns. Such deficits were most pronounced for stationary test 
patterns at low spatial frequencies in schizotypic individuals with a propensity towards unusual experiences and cognitive disorganisation. We also found reduced contrast sensitivity at high temporal frequencies $(8 \mathrm{~Hz})$ in those with a propensity towards unusual experiences. That reduced contrast sensitivity is evident in schizotypy is commensurate with the majority of findings in schizophrenics for whom reduced contrast sensitivity has been documented (although see Table 1 for variations between studies). In a broader sense, the findings presented here lend weight to the notion that schizotypy provides a useful construct for understanding the expression of psychopathology in schizophrenia.

\section{Compliance with ethical standards}

Conflict of interest The authors confirm that there are no conflicts of interest.

Open Access This article is distributed under the terms of the Creative Commons Attribution 4.0 International License (http://creativeco mmons.org/licenses/by/4.0/), which permits unrestricted use, distribution, and reproduction in any medium, provided you give appropriate credit to the original author(s) and the source, provide a link to the Creative Commons license, and indicate if changes were made.

\section{References}

Ban TA (2004) Neuropsychopharmacology and the genetics of schizophrenia: a history of the diagnosis of schizophrenia. Prog Neuropsychopharmacol Biol Psychiatry 28(5):753-762

Barch DM, Ceaser A (2012) Cognition in schizophrenia core psychological and neural mechanisms. Trends Cogn Sci. https:// doi.org/10.1016/j.tics.2011.11.015

Barrantes-Vidal N, Grant P, Kwapil TR (2015) The role of schizotypy in the study of the etiology of schizophrenia spectrum disorders. Schizophr Bull 41:S408-S416

Bodis-Wollner I (1990) Visual deficits related to dopamine deficiency in experimental animals and Parkinson's disease patients. Trends Neurosci 13:296-307

Bora E, Baysan Arabaci L (2009) Effect of age and gender on schizotypal personality traits in the normal population. Psychiatry Clin Neurosci 63(5):663-669

Brandies R, Yehuda S (2008) The possible role of retinal dopaminergic system in visual performance. Neurosci Biobehav Rev 32(4):611-656

Butler PD, Zemon V, Schechter I, Saperstein A, Hoptman M, Lim K, Revheim N, Silipo G, Javitt D (2005) Early-stage visual processing and cortical amplification deficits in schizophrenia. Arch Gen Psychiatry 62(5):495-504

Butler PD, Silverstein SM, Dakin SC (2008) Visual perception and its impairment in schizophrenia. Biol Psychiatry 64(1):40-47

Butler PD, Abeles IY, Weiskopf NG, Tambini A, Jalbrzikowski M, Legatt ME, Zemon V, Loughead J, Gur RC, Javitt DC (2009) Sensory contributions to impaired emotion processing in schizophrenia. Schizophr Bull 35(6):1095-1107. https://doi. org/10.1093/schbul/sbp 109

Cadenhead KS, Dobkins K, McGovern J, Shafer K (2013) Schizophrenia spectrum participants have reduced visual contrast sensitivity to chromatic (red/green) and luminance (light/dark) stimuli: new insights into information processing, visual channel function, and antipsychotic effects. Front Psychol. 4:535. https://doi.org/10.3389/fpsyg.2013.00535

Calderone DJ, Martinez A, Zemon V, Hoptman MJ, Hu G, Watkins JE, Javitt DC, Butler PD (2013) Comparison of psychophysical, electrophysiological, and fMRI assessment of visual contrast responses in patients with schizophrenia. NeuroImage. 67:153-162

Chen Y, Palafox GP, Nakayama K, Levy DL, Matthysse S, Holzman PS (1999) Motion perception in schizophrenia. Arch Gen Psychiatry 56(2):149-154

Chen Y, Levy DL, Sheremata S, Nakayama K, Matthysse S, Holzman PS (2003) Effects of typical, atypical, and no antipsychotic drugs on visual contrast detection in schizophrenia. Am J Psychiatry 160:1795-1801

Chen Y, Norton D, McBain R (2014) Effects of domain-specific noise on visual motion processing in schizophrenia. PLoS One 9(6): 999031

Cimmer C, Szendi I, Csifcsák G, Szekeres G, Ambrus Kovács Z, Somogyi I, Benedek G, Janka Z, Kéri S (2006) Abnormal neurological signs, visual contrast sensitivity, and the deficit syndrome of schizophrenia. Prog Neuropsychopharmacol Biol Psychiatry 30(7):1225-1230

Djamgoz MB, Hankins MW, Hirano J, Archer SN (1997) Neurobiology of retinal dopamine in relation to degenerative states of the tissue. Vision Res 37:3509-3529

Ettinger U, Mohr C, Gooding DC, Cohen AS, Rapp A, Haenschel C, Park S (2015) Cognition and brain function in schizotypy: a selective review. Schizophr Bull 41:S417-S426

Fonseca-Pedrero E, Lemos-Giráldez S, Muñiz J, García-Cueto E, Campillo-Álvarez A (2008) Schizotypy in adolescence: the role of gender and age. J Nerv Ment Dis 196(2):161-165

Franzek E, Beckmann H (1998) Different genetic background of schizophrenia spectrum psychoses: a twin study. Am J Psychiatry 155(1):76-83

Harvey PD, Bowie CR, Friedman JI (2001) Cognition in schizophrenia. Curr Psychiatry Rep 3:423-428

Howes OD, McCutcheon R, Owen MJ, Murray RM (2017) The role of genes, stress, and dopamine in the development of schizophrenia. Biol Psychiatry 81(1):9-20

Jacob SN, Nienborg H (2018) Monoaminergic neuromodulation of sensory processing. Front Neural Circuits. 10(12):51. https:// doi.org/10.3389/fncir.2018.00051

Keri S, Antal A, Szekeres G, Benedek G, Janka Z (2002) Spatiotemporal visual processing in schizophrenia. J Neuropsychiatry Clin Neurosci 14:190-196

Lenzenweger MF (2018) Schizotypy, schizotypic psychopathology and schizophrenia. World Psychiatry. 17(1):25-26

Luck SJ, Gold JM (2008) The construct of attention in schizophrenia. Biol Psychiatry 64(1):34-39

Mason M, Claridge G (2006) The oxford-liverpool inventory of feelings and experiences (O-LIFE): further description and extended norms. Schizophr Res 82:203-211

Masson G, Mestre D, Blin O (1993) Dopaminergic modulation of visual sensitivity in man. Fundam Clin Pharmacol 7(8):449-463

Mata I, Matais-Colx D, Peralta V (2005) Schizotypal personality questionnaire-brief: factor structure and influence of sex and age in a nonclinical population. Pers. Individ. Diff. 38:1183-1192

Meehl PE (1962) Schizotaxia, schizotypy, schizophrenia. Am Psychol 17(12):827-838

Ming W, Palidis DJ, Spering M, McKeown MJ (2016) Visual contrast sensitivity in early-stage parkinson's disease. Invest Ophthalmol Vis Sci 57(13):5696-5704

Owsley C (2003) Contrast sensitivity. Ophthalmol Clin North Am. $16: 171-177$ 
Ridder A, Müller ML, Kotagal V, Frey KA, Albin RL, Bohnen NI (2017) Impaired contrast sensitivity is associated with more severe cognitive impairment in Parkinson disease. Parkinsonism Relat Disord. 34:15-19

Samani N, Proudlock F, Siram V, Suraweera C, Hutchinson C, Nelson C, Al-Uzri M, Gottlob I (2018) Retinal layer abnormalities as biomarkers of schizophrenia. Schizophr Bull 44(4):876-885

Shoshina I, Shelepin Y, Semenova NB (2014) Frequency-CS of visual stimulus perception in patients with Schizophrenia treated with atypical and typical antipsychotics. Human Physiol 40:35-39

Slaghuis WL (1998) Contrast sensitivity for stationary and drifting spatial frequency gratings in positive- and negative-symptom schizophrenia. J Abnorm Psychol 107:49-62

Slaghuis WL (2004) Spatio-temporal luminance contrast sensitivity and visual backward masking in schizophrenia. Exp Brain Res 156:196-211
Tagliati M, Bodis-Wollner I, Kovanecs I, Stanzione P (1994) Spatial frequency tuning of the monkey pattern ERG depends on D2 receptor-linked action of dopamine. Vision Res 34:2051-2057

Yang AC, Tsai SJ (2017) New targets for schizophrenia treatment beyond the dopamine hypothesis. Int J Mol Sci 18(8):E1689. https ://doi.org/10.3390/ijms18081689

Publisher's Note Springer Nature remains neutral with regard to jurisdictional claims in published maps and institutional affiliations. 